ARTICLE

Received 7 Feb 2016 | Accepted 25 Aug 2016 | Published 30 Sep 2016

\title{
Acoustic omni meta-atom for decoupled access to all octants of a wave parameter space
}

Sukmo Koo ${ }^{1, \dagger}$, Choonlae Cho ${ }^{1}$, Jun-ho Jeong ${ }^{2} \&$ Namkyoo Park ${ }^{1}$

The common behaviour of a wave is determined by wave parameters of its medium, which are generally associated with the characteristic oscillations of its corresponding elementary particles. In the context of metamaterials, the decoupled excitation of these fundamental oscillations would provide an ideal platform for top-down and reconfigurable access to the entire constitutive parameter space; however, this has remained as a conceivable problem that must be accomplished, after being pointed out by Pendry. Here by focusing on acoustic metamaterials, we achieve the decoupling of density $\rho$, modulus $B^{-1}$ and bianisotropy $\xi$, by separating the paths of particle momentum to conform to the characteristic oscillations of each macroscopic wave parameter. Independent access to all octants of wave parameter space $\left(\rho, B^{-1}, \xi\right)=(+/-,+/-,+/-)$ is thus realized using a single platform that we call an omni meta-atom; as a building block that achieves top-down access to the target properties of metamaterials.

\footnotetext{
${ }^{1}$ Photonic Systems Laboratory, Department of Electrical and Computer Engineering, Seoul National University, Seoul 08826, Korea. ${ }^{2}$ Department of Nano Manufacturing Technology, Korea Institute of Machinery and Materials, Daejeon 34103, Korea. †Present address: Moore Laboratory, Department of Medical Engineering, California Institute of Technology, Pasadena, California 91125, USA. Correspondence and requests for materials should be addressed to N.P. (email: nkpark@snu.ac.kr).
} 
$\mathrm{T}$ he general features of wave propagation are ultimately determined by the properties of its medium, where the wave travels through. In order to achieve an extreme manipulation of wave propagation, the accessibility to the unusual space of wave parameters is, therefore, obligatory. A wide variety of extreme wave parameters and their applications have been realized for different waves and material systems in the context of metamaterials; spanning the fields of, acoustics ${ }^{1-15}$, photonics ${ }^{16-37}$, thermodynamics ${ }^{38}$, elasto-dynamic $3^{39-41}$, seismics ${ }^{42}$, among others. Negative- ${ }^{1-4,16-18,39}$, zero- $^{5-7,19-21}$, ultrahigh-index ${ }^{22}$, hyperbolic-23, anisotropic- ${ }^{8,9,24}$, bianisotropic- ${ }^{24-26}$, chiral- ${ }^{16,24}$ and disordered-metamaterials ${ }^{27}$ have been demonstrated, along with their applications towards cloaking ${ }^{19,28,29}$, super-focusing, perfect absorption ${ }^{10}$, iso-spectrality ${ }^{27}$, meta-surface hologram ${ }^{30}$ and frequency-agile memory ${ }^{31}$.

With keen interest on applications, reconfigurable control of wave parameters has also become main stream in wave physics ${ }^{28,32,33}$. Nonetheless, although the decoupling of fundamental wave parameters has been envisaged as an ideal platform towards the top-down and deterministic reconfiguration of the meta-atom (Pendry et al. ${ }^{28}$ ), its feasibility has remained merely as a plausible idea that has yet to be proven. In most cases, the decoupling of constituent parameters has been achieved via the combination of elementary resonators in a non-isotropic and polarization-dependent form ${ }^{17,18,29,39}$. At present, strategies for metamaterial design have been based on bottom-up approaches; in which building blocks are proposed first, and subsequent design is performed iteratively until a specific index and impedance are achieved. Although it has been recently reported that pentamode metamaterials can provide decoupled access to all positive mechanical wave parameters ${ }^{40}$, achieving full accessibility to the entire space of wave parameters with the designs of existing metamaterials remains an open question, and the existence of an omnipotent meta-atom platform for reconfigurable and seamless access to the wave parameter space also has yet to be answered.

Inspired by the fundamental oscillations of the elementary particle of a wave, in this communication we propose an entirely new design strategy for the meta-atom. Focusing on an acoustic platform, the criteria for the decoupling of wave parameters are derived from first principles, and an omni meta-atom that achieves independent, broad-range access to all octants of the wave parameter space $\left(\rho, B^{-1}, \xi\right)$ is demonstrated. Based on the top-down access capability for target $\left(\rho, B^{-1}, \xi\right)$, we then demonstrate a new class of meta-devices; bianisotropic meta-surfaces for independent beam shaping of transmissionand reflection-waves, and as well as zero-index waveguides for pressure-velocity conversion. Our work provides a deeper insight on the relationship between wave parameters and the internal structures of a meta-atom, and paves a new route towards systematic access to target wave parameters.

\section{Results}

First-principle derivations of effective wave parameters. Understanding that the electromagnetic wave parameters $\varepsilon$ and $\mu$ of a classical atom are directly related to the linear- and angular-oscillations of an electron respectively, the insight of this study begins from the characteristic oscillation of elementary particles, in relation to wave parameters of interest. In this respect, the derivation of effective parameters for an acoustic wave ( $\rho_{x}$ : density for $x$ direction, $B$ : bulk modulus) from the characteristic motions of acoustic particles is straightforward (Fig. 1a).

Based on the duality between electromagnetic and acoustic waves, ${ }^{5,11}$, we first modify Alù's derivation of electromagnetic macroscopic wave parameters ${ }^{34}$, to derive effective parameters of an acoustic system from first principles (details in Supplementary Note 1). In the limit of a long wavelength ( $|\boldsymbol{\beta}| a<<\pi, \boldsymbol{\beta}$ : effective wavevector, $a$ : lattice constant), $B^{-1}$ and $\rho_{x}$ are then expressed as,

$$
\begin{gathered}
B^{-1} \sim \frac{\int_{S} B_{s}^{-1} p d S}{\int_{S} p d S+\frac{i \omega}{2} \int_{S} r\left(\rho_{s r}-1\right) v_{r} d S}, \\
\rho_{x} \sim \frac{\int_{S} \rho_{s x} v_{x} d S}{\int_{S} v_{x} d S-\frac{i \omega}{2} \int_{S}\left(B_{s}^{-1}-1\right) p x d S}
\end{gathered}
$$

for a two-dimensional unit cell $S$; with a distributed particle density tensor $\rho_{s}$ (subscripts $x, r$ denote density directions) and modulus $B_{s}$ (normalized to air) of the constituting materials inside $S$, where $\mathbf{r}$ is the position vector measured from the cell centre, and $p$ and $\mathbf{v}$ each correspond to the pressure and velocity fields at $\mathbf{r}$.

Design criteria for the decoupling of wave parameters. Important to note from equation (1) is the presence of crosscoupling terms in the denominators of $B^{-1}\left(\rho_{s r}\right)$ and $\rho_{x}\left(B_{s}^{-1}\right)$, which hinder the decoupled access to $B^{-1}$ and $\rho_{x}$. Out of various possibilities, we try to spatially decouple $B^{-1}$ and $\rho_{x}$, by employing a meta-atom with an inner sub-cell (IS) of radial symmetry and outer sub-cells (OS) of decoupled $x, y$ linear vibrations, each conforming to the fundamental oscillations of $B^{-1}$ and $\rho_{x, y}$ (Fig. 1a); in a square lattice composed of a membrane, air and solid walls (Fig. 1b).

Under these settings, the expansion near the Dirac point ${ }^{5,6,19}$ of zero compressibility and zero density constrain the movement of outer- and inner-membranes, enabling further reduction of the equations; radial movement of the outer membrane is prohibited (as $B^{-1} \sim 0$ ), and outer- and inner-membrane should move out of phase but with the same momentum value (as $\rho_{x} \sim 0$ ). In this case, by employing a heavy mass for $\rho_{\mathrm{m}}$ (or a large thickness for the inner membrane), equation (1) is reduced to (details in

a
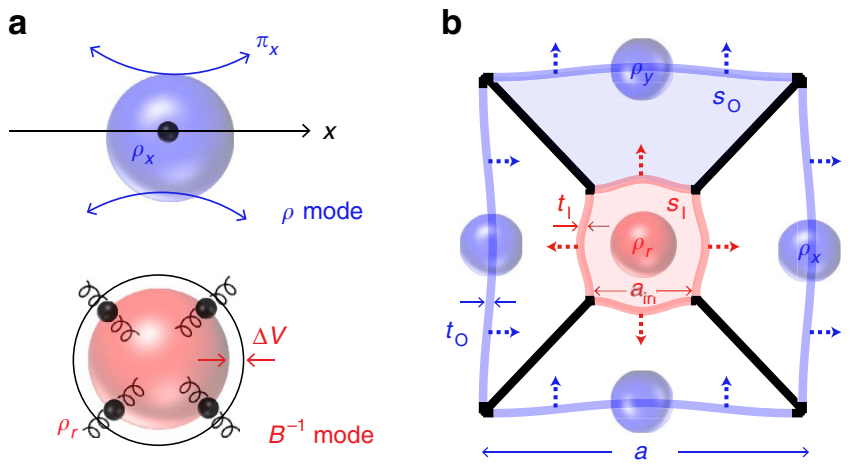

Figure 1 | Characteristic oscillations of acoustic atoms and the decoupling of constitutive wave parameters. (a) Linear and radial characteristic oscillations of acoustic atoms for $\rho$ and $B^{-1}$, respectively. (b) Schematic of the proposed meta-atom. blue and red: outer and inner membranes; black: solid wall; $t_{\mathrm{O}(\mathrm{I})}$ : outer (inner) membrane thickness; $s_{\mathrm{O}}$ (shaded in light blue) and $s_{I}$ (shaded in light red): outer and inner area (region) in the meta-atom unit cell. 
Supplementary Note 2),

$$
\begin{gathered}
B^{-1}=\frac{\int_{S 0} B_{s 0}^{-1} p d S}{\frac{i \omega}{2} \int_{I S \mathrm{~m}} r \rho_{\mathrm{m} r} v_{r} d S}, \\
\rho_{x}=\frac{\int_{I S \mathrm{~m}} \rho_{\mathrm{m} x} v_{x} d S+\int_{O S \mathrm{~m}} \rho_{\mathrm{m} x} v_{x} d S}{\int_{O S 0} v_{x} d S},
\end{gathered}
$$

a

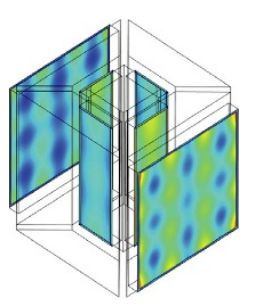

C

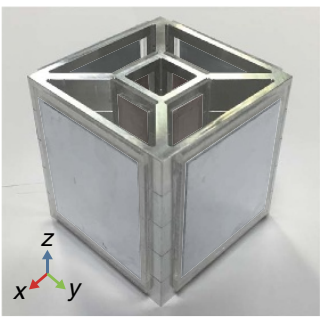

e

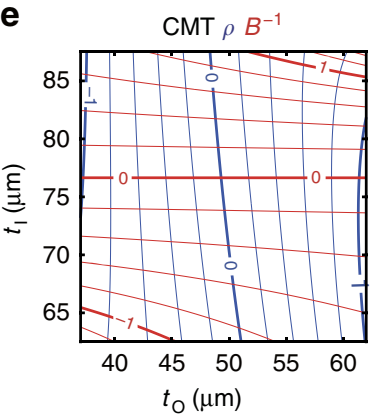

g

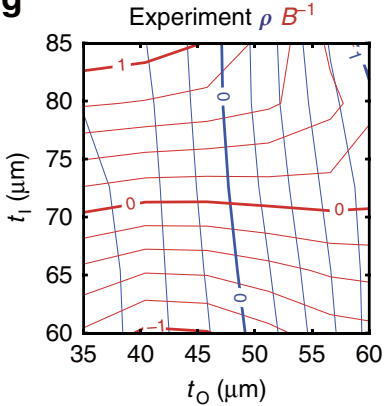

i

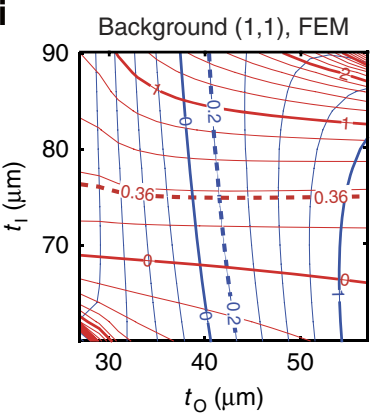

d b
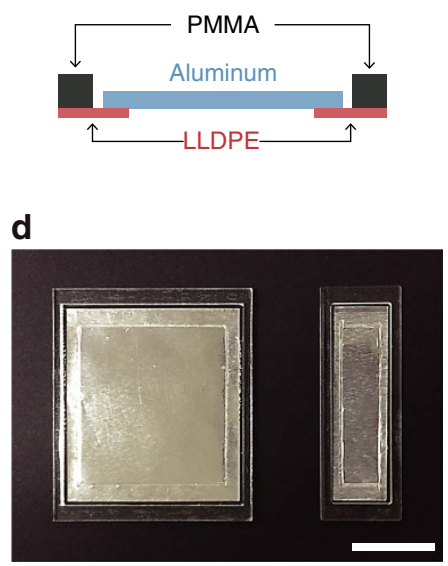

$\mathbf{f}$

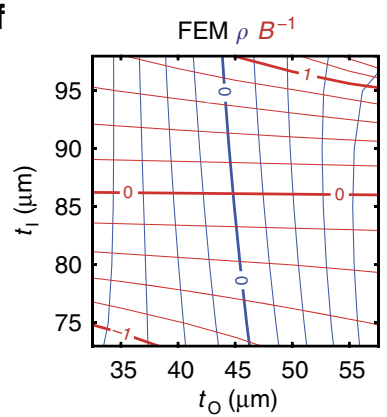

h

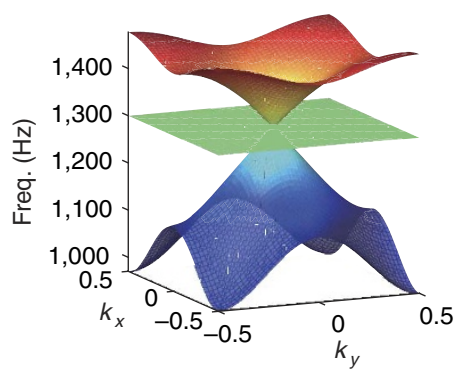

j

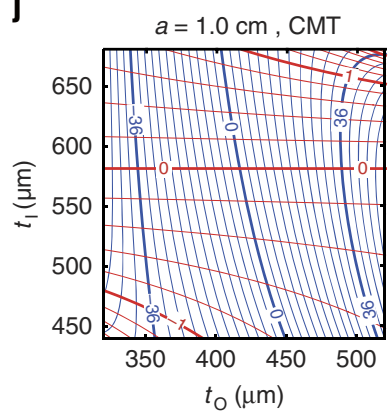

where subscript $\mathrm{m}$ and 0 denote the material (membrane and air) for the given physical quantities (for example, $\rho, B^{-1}$ ) at $S$, IS and OS. Most importantly, equation (2) shows the direct control of effective $B^{-1}$ with mass $\rho_{\mathrm{m}}$ of the inner-membrane in the denominator, which justifies the proposed approach of dividing the meta-atom into the inner- and outer-sub-cells that correspond to the fundamental oscillations of $B^{-1}$ and $\rho_{x}$. With the inner membrane mass $\rho_{\mathrm{m}}$ determined for $B^{-1} \sim 0$, then the control of effective $\rho_{x}$ with the tuning of only outer-membrane mass (that is, second term $\rho_{\mathrm{m} x}$ in the numerator of $\rho_{x}$ ) is consequently realized.

A more explicit solution for the structure shown in Fig. 1b can be obtained by using the coupled mode theory (CMT). Applying Newton's law to the membranes and Hooke's law to the air region, the decoupled relation for $\rho$ (proportional to outer membrane mass $\rho_{\mathrm{Al}} t_{\mathrm{O}}$ ) and $B^{-1}$ (proportional to inner membrane mass $\rho_{\mathrm{Al}} t_{\mathrm{I}}$ ) are again confirmed in the long-wavelength limit, as shown in equation (3) (derivation in Supplementary Note 3, see also Supplementary Fig. 1):

$$
\begin{aligned}
& \rho_{0} \rho=\frac{\rho_{\mathrm{Al}} t_{\mathrm{O}}}{a}-\frac{B_{0}}{s_{\mathrm{O}}}\left(2+\frac{s_{\mathrm{I}}}{s_{\mathrm{O}}}\right) \frac{1}{\omega^{2}} \\
& \left(\frac{a}{2} \cdot \frac{B_{0}}{s_{\mathrm{O}}}\right)^{2} B^{-1}=\frac{\rho_{\mathrm{Al}} t_{\mathrm{I}}}{a_{\mathrm{in}}} \omega^{2}-\frac{B_{0}}{s_{\mathrm{O}}}\left(1+\frac{4 s_{\mathrm{O}}}{s_{\mathrm{I}}}\right) .
\end{aligned}
$$

where $\omega$ being the angular frequency, $\rho_{0}$ and $\rho_{\mathrm{Al}}$ being the density of air and Al membrane, $a$ being the lattice constant, $a_{\text {in }}$ being the size of the IS, $t$ being the membrane thickness and $s$ denotes the area of inner- (I) and outer- (O) sub-cells, respectively. Worth to mention, with equation (3), it is also possible to achieve independent control of $\left(\rho, B^{-1}\right)$ as a function of pressures $\left(\sim B_{0}\right)$ or volumes/areas $\left(\sim s_{\mathrm{O}}, s_{\mathrm{I}}\right)$ in sub-cells.

Demonstration of the decoupling of effective parameters. The meta-atom's membrane motion calculated by the finite element method (FEM using commercially available program COMSOL), and the schematic of the membrane are shown in Fig. 2a,b, respectively. Experimental realizations of the meta-atom and membranes are shown in Fig. 2c,d. Details of the structure and material parameters are described in the Methods section. Using the un-approximated CMT solutions in Supplementary Eq. (14),

Figure 2 | Experimental realization of the meta-atom and the decoupling of effective wave parameters. (a) Deflection pattern of the membrane obtained from three-dimensional FEM. (b) Structure of the membrane. $(\mathbf{c}, \mathbf{d})$ Three-dimensional experimental realization of the meta-atom and membrane (white line denote scale bar, $20 \mathrm{~mm}$ ). (e) CMT (f) twodimensional FEM and ( $\mathbf{g}$ ) experimental results showing decoupled access to $\rho$ (blue lines) and $B^{-1}$ (red lines) with outer and inner membranes thicknesses $t_{\mathrm{O}}$ and $t_{1}$, respectively. Except (j) grid spacings for $\rho$ (blue lines) and $B^{-1}$ (red lines) are 0.2. The thickness of the Al wall in the FEM and experiment is set at $3 \mathrm{~mm}$. The lattice constant and height of the cell were 6 and $7 \mathrm{~cm}$, respectively. In the experiment, measurements were taken with a membrane thickness resolution at $10 \mu \mathrm{m}$. (h) CMT obtained dispersion relation of the meta-atom, with the design parameter of $\left(t_{\mathrm{O}}=49.3 \mu \mathrm{m}\right.$, $\left.t_{1}=76.6 \mu \mathrm{m}\right)$. (i) Shifting the centre of the decoupling operation to $\left(\rho_{\mathrm{c}}\right.$ $\left.B_{c}^{-1}\right)=(0.2,0.36)$ (intersection of the thick dashed lines), with the effective medium approach (placing the meta-atom in a host medium of air. see Supplementary Fig. 4 and Supplementary Note 5). (j) Extension of density tuning range $(-36<\rho<36)$ with the use of smaller unit cell $(a=1 \mathrm{~cm})$ having heavier mass plates (see Supplementary Fig. 5 and Supplementary Note 5). Grid spacings for $\rho$ and $B^{-1}$ for $\mathbf{j}$ are 3 and 0.2 . Operation frequency is $1,300 \mathrm{~Hz}$, for all cases. 
in Fig. 2e we visualize the mapping of $\left(\rho, B^{-1}\right)$ in terms of membrane thickness of outer- and inner-sub-cell $\left(t_{\mathrm{O}}, t_{\mathrm{I}}\right)$ at $1,300 \mathrm{~Hz}$, near the Dirac point ${ }^{5,6,19}$ (Fig. $2 \mathrm{~h}$ and Supplementary Fig. 2 in Supplementary Note 3). From Fig. 2e, perfectly orthogonal decoupling between $\rho$ and $B^{-1}$ is evident, especially near $\left(\rho, B^{-1}\right)=(0,0)$, which is in excellent agreement with FEM and experimental results (Fig. 2f,g). The membrane movements of $\left(\rho, B^{-1}\right)$ modes exhibit dipolar- and monopolar- patterns, confirming the proposed ansatzs and discussions regarding Fig. 1 and equation (2) (see Supplementary Note 4 and Supplementary Fig. 3). It is important to note that, inverse determination of the meta-atom structural parameter $\left(t_{\mathrm{O}}, t_{\mathrm{I}}\right)$ is also possible from the target $\left(\rho, B^{-1}\right)$ values using equation (3) or Fig. 2e. Finally, we also show decoupled operation away from the Dirac point (Fig. 2i) and extension of the tuning range (Fig. 2j), with the use of effective medium approach and smaller unit cell of heavier membranes, respectively (Supplementary Note 5 and Supplementary Figs 4 and 5).

Decoupled implementation of acoustic bianisotropy. The decoupling could be generalized to include the other wave parameter axis of bianisotropy ${ }^{24-26}$. Meanwhile the bianisotropy $\xi$ has been demonstrated using $\Omega$-type metamaterials ${ }^{25,26}$ in nanophotonics, yet it needs to be conceptualized and demonstrated for acoustic metamaterials. In parallel to electromagnetic bianisotropy that exchanges kinetic and potential energies (or equivalently, electric and magnetic fields), here we investigate the coupling constant $\xi$ that connects the velocity and the pressure field (Fig. 3a). Considering that $\xi$ is related to structural asymmetry ${ }^{34}$, we choose to apply asymmetry in the form of $t_{\mathrm{I}} \pm \Delta t_{\mathrm{I}} / 2$, in order to create non-zero $\xi$ (Fig. 3b,c). The analytically derived $\xi$ (Supplementary Eq. (17) in Supplementary Note 6; its approximation in equation (4)) shows a linear relation with $\Delta t_{\mathrm{I}}$, independently from $t_{\mathrm{I}}$ or $t_{\mathrm{O}}$ (Fig. $3 \mathrm{~d}$ and Supplementary Fig. 6). Therefore, near-perfect decoupling between $\rho, B^{-1}$ and $\xi$ near $(0,0,0)$ (Fig. $3 e, f)$ is realized, in excellent agreement with the numerical and experimental results.

$$
\xi=-\frac{Z_{0} s_{\mathrm{I}} \omega \rho_{\mathrm{Al}}}{2 a a_{\mathrm{in}}} \Delta t_{\mathrm{I}}
$$

Asymmetric impedance manipulation based on the bianisotropy. The salient feature of the bianisotropic medium is in the asymmetric impedance manipulation of the wave with the exchange in kinetic and potential energy during wave propagation. Using the bianisotropic meta-atom at a matched zero index, here we report a perfect transmission between over- $\lambda$ and sub- $\lambda$ widths (or impedances) of waveguides (Fig. $3 \mathrm{~g}$ ); achieving super-funnelling/radiation above the $\lambda$-zone limit ${ }^{43,44}$.

As shown in Fig. 3g, six bianisotropic zero index meta-atoms $\left(\xi=-0.158\right.$ and $\left.\rho=B^{-1}=0\right)$ in the output waveguide, in addition to a layer of meta-atoms of $\left(\rho, B^{-1}, \xi\right)=(0,0,0)$ in the input side are used. The non-zero $\xi=-0.158$ value for ideal exponential field evolution and complete impedance conversion is calculated from the ratio of input/output waveguide widths and the number of bianisotropic meta-atoms $(\xi=\log$ $\left(w_{1} / w_{2}\right) /\left(2 k_{0} \cdot 6 a\right)$; see Supplementary Note 7 and Supplementary Fig. 7). Achieving exact $\left(\rho, B^{-1}, \xi\right)$ values for the meta-atoms from the independent control of $\left(t_{\mathrm{O}}, t_{\mathrm{I}}, \Delta t_{\mathrm{I}}\right)$, Fig. $3 \mathrm{~g}$ shows the pressure field calculated by the FEM, exhibiting the super-funnelling from over- $\lambda$ to the sub- $\lambda$ width waveguide $\left(w_{1} / \lambda=3.4\right.$ to $\left.w_{2} / \lambda=0.227, w_{1} / w_{2}=15\right)$. Reflectionless, ideal impedance conversion with the bianisotropic zero-index waveguide is clear; in addition to the suppression of higher order mode excitation from the meta-atom array of matched zero index. Excellent agreement with analytical result (Supplementary Eq. (24) in Supplementary Note 8) for an extreme case of a single meta-atom for $\left(w_{1} / w_{2}\right)=2$ are also demonstrated experimentally (Fig. 3h).

Bianisotropic meta-surface for decoupled $T$ and $R$ wavefront. As a final application example supported by the capability of independently addressing target $\rho, B^{-1}$, and also $\xi$ values, we demonstrate a bianisotropic wave front shaping in a metasurface $^{12,13,30,35-37}$ context, of critical novelty in a transmission-reflection ( $\mathrm{T}$ and $\mathrm{R}$ ) decoupled form. Under the notion of the generalized Snell's law ${ }^{35}$, we emphasize that the transmission- and reflection-decoupled bianisotropic wave front shaping can be achieved only via independent control of $\left(\phi_{\mathrm{R}}, \phi_{\mathrm{T}}\right)$ at the meta-surface; where the controllability of $\xi$ for individual metaatom has a critical role in achieving $n_{\mathrm{R}} \neq n_{\mathrm{T}}$ while maintaining the same value of $n_{\text {eff }}$ over the entire surface.

To realize independent controllability of $\left(\phi_{\mathrm{R}}, \phi_{\mathrm{T}}\right)$, in Fig. 4a we plot the phase shift contour $\left(\phi_{\mathrm{R}}, \phi_{\mathrm{T}}\right)$ in the parameter octant space of $\left(\rho, B^{-1}, \xi\right)$, achieving 50:50 power division for the transmitted and reflected waves (details in Supplementary Note 9 and Supplementary Fig. 8). It is stressed that, in the absence of bianisotropy $(\xi=0)$, it is impossible to adjust $\left(\phi_{\mathrm{R}}, \phi_{\mathrm{T}}\right)$ under the given 50:50 power splitting condition, as evident from Fig. 4a. From target phase shifts $\left(\phi_{\mathrm{R}}, \phi_{\mathrm{T}}\right)$ of an individual meta-atom (in a $40 \times 1$ array), calculations of $\left(\rho, B^{-1}, \xi\right)$ are obtained from Supplementary Eq. (25) (details in Supplementary Note 9 and Supplementary Fig. 9), which achieves ordinary $(\Delta \phi(x)=0)$ or anomalous $(\Delta \phi(x) \neq 0)$ transmission and reflections (Supplementary Fig. 10). Subsequent, top-down determination of the corresponding $\left(t_{\mathrm{O}}, t_{\mathrm{I}}, \Delta t_{\mathrm{I}}\right)$ from $\left(\rho, B^{-1}, \xi\right)$ is then straightforward. Independent control of the reflected wave, the transmitted wave, as well as the simultaneous control of the reflected- and transmitted-wave compared with the reference (left figure), with corresponding phase maps at the bottom of the figures are shown in Fig. $4 \mathrm{~b}$.

Experimental realization of a bianisotropic meta-surface has also been carried out using a $10 \times 1$ meta-atom array, in a $70 \times 150 \times 7 \mathrm{~cm}$ box with an acoustic absorber and an $8 \times 1$ speaker array (Fig. 4c). With the finite dimension of the set-up used in this study, experiment has been performed with an incidence wave normal to the meta-atom array. Figure $4 \mathrm{~d}$ shows scattered pressure field patterns together with the reflection- and transmission-phase $\left(\phi_{\mathrm{R}}, \phi_{\mathrm{T}}\right)$ of individual meta-atoms; dotted lines from the target design, square marks from the impedance tube measurements, and solid lines from the pressure field scanning measurements. With precise access to $\left(\phi_{\mathrm{R}}, \phi_{\mathrm{T}}\right)$ values from the control of $\left(\rho, B^{-1}, \xi\right)$ in each meta-atom, decoupled manipulation of the reflection- and transmission-wave fronts are successfully realized experimentally.

\section{Discussion}

In summary, with the insight gained from fundamental oscillations of the wave supported by first principles of homogenization theory, we demonstrated an acoustic omni meta-atom that achieves decoupled access to the target waveparameter in the octant space of $\left(\rho, B^{-1}, \xi\right)$, with the tuning of structural factors of the meta-atom $\left(t_{\mathrm{O}}, t_{\mathrm{I}}, \Delta t_{\mathrm{I}}\right)$. Excellent agreements between CMT-based solutions, FEM-based numerical analysis and experiments have been realized, confirming the top-down design capability that addresses target $\left(\rho, B^{-1}, \xi\right)$ values. Shifting the centre of decoupling operation in the frame of effective medium theory, as well as the extension of effective parameter tuning range have been verified numerically, in 

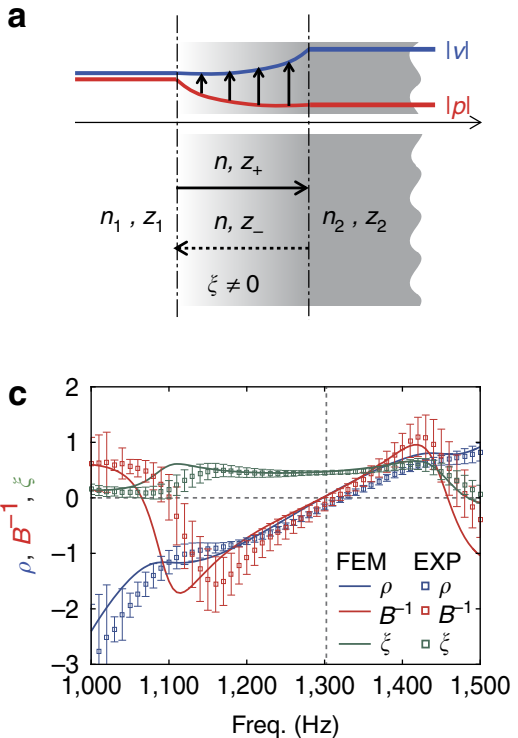

e

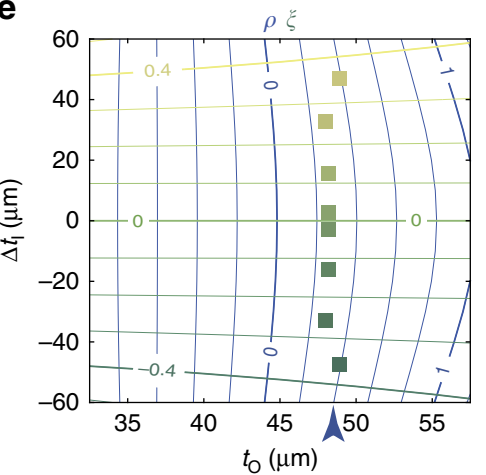

g

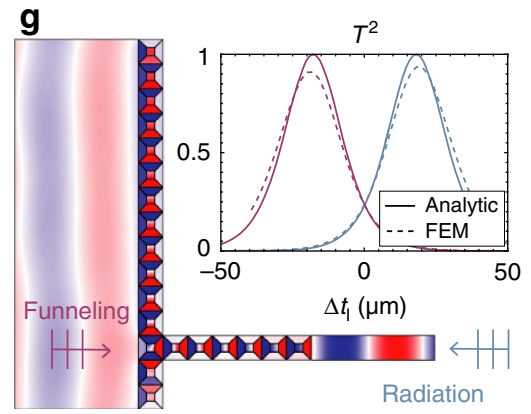

b
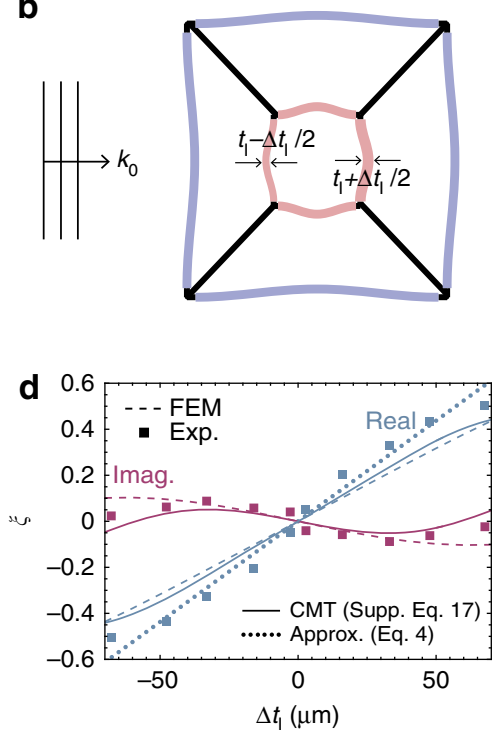

$\mathbf{f}$

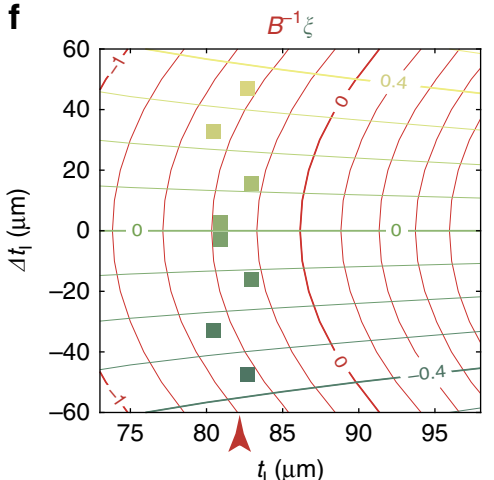

h

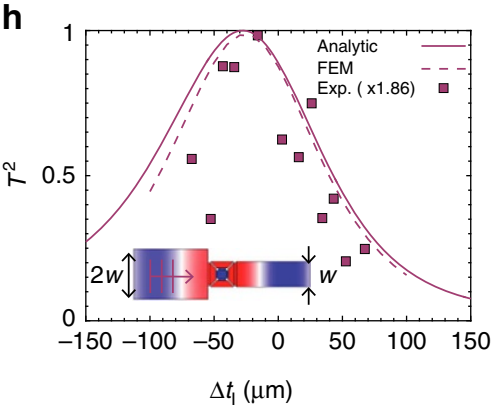

Figure 3 | Implementation of acoustic bianisotropy and bianisotropic meta-waveguide. (a) Exchange of velocity- and pressure-field amplitude (kinetic and potential energies) in an acoustic bianisotropic medium. (b) Schematic of the bianisotropic meta-atom with an asymmetric arrangement of the membrane thickness. (c) FEM calculated, and experimentally measured wave-parameter spectra of the bianisotropic meta-atom, designed to achieve $\left(\rho, B^{-1}, \xi\right)=(0,0,0.5)$ at $1,300 \mathrm{~Hz}$. The zero density and zero compressibility at the operation frequency of $1,300 \mathrm{~Hz}$ are the result of zero crossing between two nearby resonances $\left(\sim 1,100\right.$ and $1,400 \mathrm{~Hz}$ ). (d) Tuning of $\xi$ with $\Delta t_{1}$ from the experiment (square symbols), CMT (solid line), FEM (dotted lines), and the approximation (point symbols, equation (4)). (e,f) Tuning of $(\rho, \xi)$ and $\left(B^{-1}, \xi\right)$ with $\Delta t_{1}$. (g) FEM-calculated pressure field for the super-funnelling $\left(w_{1} / w_{2}=15, \xi=-0.158, \Delta t_{1}=-18 \mu \mathrm{m}\right)$. The inset shows the transmittance for super-funnelling and super-radiation. (h) Experimentally obtained super-funnelling with a single meta-atom at different $\Delta t_{1}$ (or bianisotropy $\xi$ ). $w_{1} / w_{2}=2$. Operation frequency is $1,300 \mathrm{~Hz}$, for all cases.

agreement with the proposed theory. The feasibility of active tunability using pressures and volumes in sub-cells has also been discussed with CMT equations. With the capability of independently- and deterministically-addressing target wave parameters, we show applications of bianisotropic pressurevelocity impedance conversion, and reflection-transmission decoupled wave front shaping. We note that, likewise most of resonance based metamaterials, the bandwidth limitation in our design is considered as an engineering subject which could be mitigated with various approaches $3,10,23$. Our work opens a new paradigm in the design of meta-atoms by overcoming difficulties observed from the bottom-up approach, and provides an ideal platform by resolving the previously envisaged but unanswered issue of the decoupled excitation of constitutive parameters. Using the same approach, we expect further extension of decoupled access to other waves (that is, ultra-sonic ${ }^{45}$, electromagnetic, elastic ${ }^{39}$ and thermal) and wave parameters (that is, stress, strain, gyrotropy and chirality). 
a

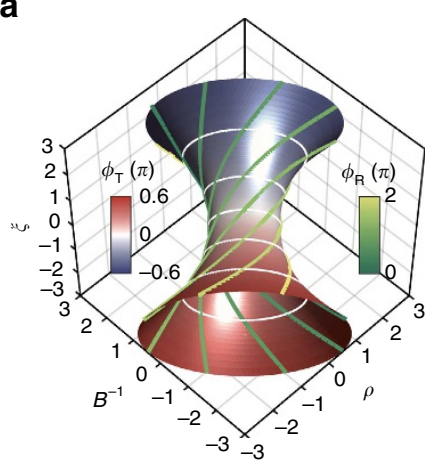

C

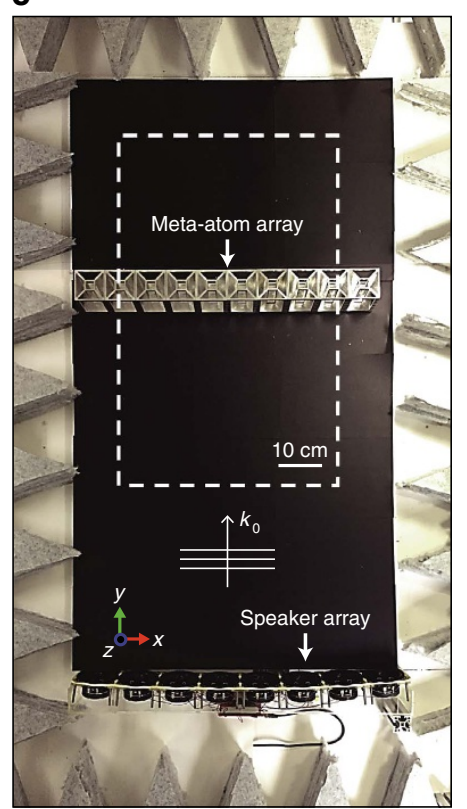

b
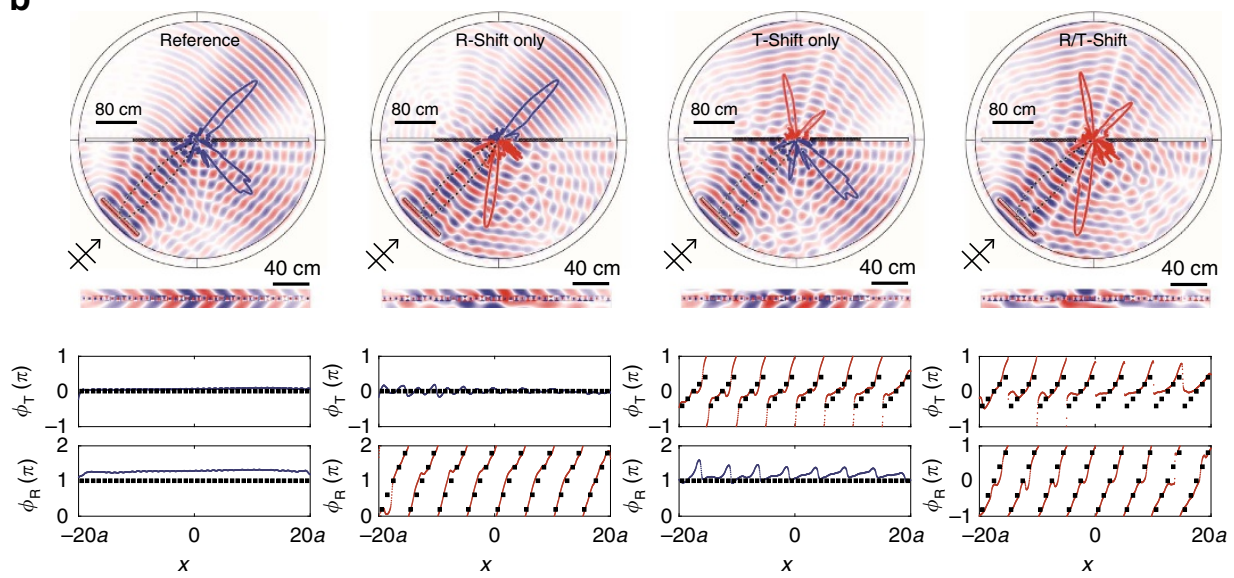

d
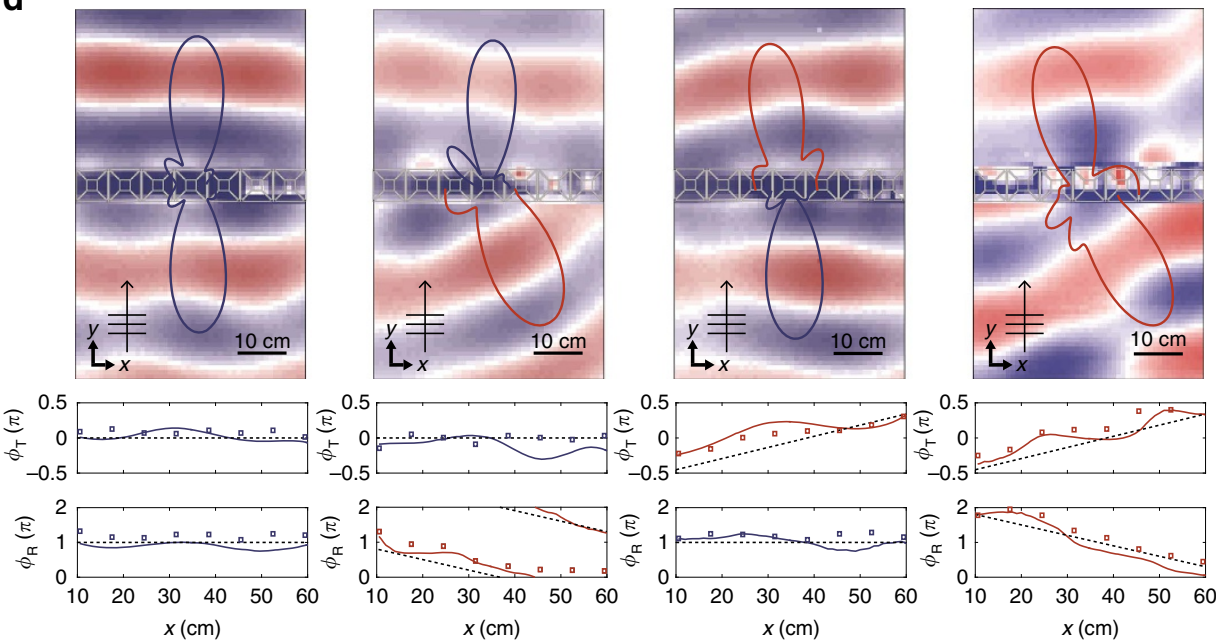

$x(\mathrm{~cm})$

$x(\mathrm{~cm})$

$x(\mathrm{~cm})$

Figure 4 | Transmission and reflection decoupled wave front shaping using a bianisotropic meta-surface. (a) Phase shift contour $\left(\phi_{\mathrm{R}}, \phi_{\mathrm{T}}\right)$ in the parameter octant space of $\left(\rho, B^{-1}, \xi\right)$ for a 50:50 power division for the transmitted and reflected waves (Supplementary Note 9). (b) FEM calculated pressure field patterns for an incidence wave from the bottom at $45^{\circ}$. Left to right: reference, shift in the reflection, transmission, and reflection and transmission wavefront. The overlaid far-field polar plots are calculated from the near-field data. Transmission and reflection phases of the meta-atom array (dotted lines are for the design; the solid lines are measured from the FEM calculations) are shown at the bottom of each figure. (c) Top view of the experimental set-up. The experiment was performed with a $10 \times 1$ meta-atom array in a $70 \times 150 \times 7 \mathrm{~cm}$ box with an acoustic absorber and an $8 \times 1$ speaker array. (d) Experimentally measured scattered pressure field patterns and calculated far-field polar plots for a normal incidence wave from the bottom. Below the field patterns, the transmission- and reflection-phases of the meta-atom array are shown. Dotted lines for design, square marks from the one-dimensional impedance tube measurements, and solid lines from the experimentally measured pressure field near the meta-surface. Operation frequency is $1,300 \mathrm{~Hz}$, for all cases.

\section{Methods}

Details of the meta-atom structure and material parameters. For the experiment, we constructed a two-dimensional slab meta-atom (height $=7 \mathrm{~cm}$ ) using an $\mathrm{Al}$ sheet-loaded linear low-density polyethylene (LLDPE) membrane and a solid $\mathrm{Al}$ wall (thickness $=3 \mathrm{~mm}, a=6 \mathrm{~cm}, a_{\text {in }}=2 \mathrm{~cm}$; see Fig. 1a). To achieve the decoupling of $\left(\rho, B^{-1}\right)$ at $1,300 \mathrm{~Hz}$, the effective thicknesses $\left(t_{\mathrm{O}}, t_{\mathrm{I}}\right)$ of the $\mathrm{Al}$ sheet have been controlled between $35-60$ and $50-90 \mu \mathrm{m}$, respectively; same thicknesses used in CMT and FEM analyses. The densities ${ }^{14,46}$ of air and Al are assumed to be 1.21 and $2,700 \mathrm{~kg} \mathrm{~m}^{-3}$. The wave parameters of interest have been calculated by using $S$ parameters extracted from a 4-point measurement impedance tube. It is noted that we employed a composite membrane constructed with an Al-sheet mounted on top of a larger frame of an LLDPE film as shown in Fig. $2 b$.

The Al-sheet has a much greater weight and stiffness compared with the LLDPE film, and provides a method of controlling the composite membrane mass with its thickness; independently of the stiffness of the composite membrane $\left(k^{-1}\right.$ composite $\left.\sim k^{-1}{ }_{\text {LLDPE }}+k^{-1}{ }_{\mathrm{Al}}\right)$ that is primarily determined by the properties of the LLDPE film ( $10 \mu \mathrm{m}$ thick). For the fine tuning of the membrane's effective thickness (that is, mass), we used 3-8 stacked layers of Al-sheets that each had a thickness of $\sim 15 \mu \mathrm{m}$ with periodically perforated disks of a $2-\mathrm{mm}$ radius. The final dimensions of the outer (inner) LLDPE film and the Al-sheet were $54 \times 60 \mathrm{~mm}$ $(18 \times 60 \mathrm{~mm})$ and $52 \times 58 \mathrm{~mm}(16 \times 58 \mathrm{~mm})$, respectively.

Data availability. All relevant data that support the findings of this study are available from the corresponding author upon request.

\section{References}

1. Liang, Z., Willatzen, M., Li, J. \& Christensen, J. Tunable acoustic double negativity metamaterial. Sci. Rep. 2, 859 (2012).

2. Yang, M., Ma, G., Yang, Z. \& Sheng, P. Coupled membranes with doubly negative mass density and bulk modulus. Phys. Rev. Lett. 110, 134301 (2013)

3. Kaina, N., Lemoult, F., Fink, M. \& Lerosey, G. Negative refractive index and acoustic superlens from multiple scattering in single negative metamaterials. Nature 525, 77-81 (2015).

4. Cheng, Y., Xu, J. Y. \& Liu, X. J. One-dimensional structured ultrasonic metamaterials with simultaneously negative dynamic density and modulus. Phys. Rev. B 77, 045134 (2008). 
5. Liu, F., Huang, X. \& Chan, C. T. Dirac cones at $\mathrm{k}=0$ in acoustic crystals and zero refractive index acoustic materials. Appl. Phys. Lett. 100, 071911 (2012).

6. Li, Y., Wu, Y. \& Mei, J. Double Dirac cones in phononic crystals. Appl. Phys. Lett. 105, 014107 (2014).

7. Gu, Y., Cheng, Y., Wang, J. \& Liu, X. Controlling sound transmission with density-near-zero acoustic membrane network. J. Appl. Phys. 118, 024505 (2015).

8. Li, J., Fok, L., Yin, X., Bartal, G. \& Zhang, X. Experimental demonstration of an acoustic magnifying hyperlens. Nat. Mater. 8, 931-934 (2009).

9. Shen, C., Xu, J., Fang, N. X. \& Jing, Y. Anisotropic complementary acoustic metamaterial for canceling out aberrating layers. Phys. Rev. X 4, 041033 (2014).

10. Mei, J. et al. Dark acoustic metamaterials as super absorbers for low-frequency sound. Nat. Commun. 3, 756 (2012).

11. Cummer, S. A. \& Schurig, D. One path to acoustic cloaking. New J. Phys. 9, 45 (2007).

12. Zhao, J., Li, B., Chen, Z. N. \& Qiu, C. W. Redirection of sound waves using acoustic metasurface. Appl. Phys. Lett. 103, 151604 (2013).

13. Tian, Y., Wei, Q., Cheng, Y., Xu, Z. \& Liu, X. Broadband manipulation of acoustic wavefronts by pentamode metasurface. Appl. Phys. Lett. 107, 221906 (2015).

14. Lee, S. H., Park, C. M., Seo, Y. M., Wang, Z. G. \& Kim, C. K. Acoustic metamaterial with negative density. Phys. Lett. A 373, 4464-4469 (2009).

15. Park, C. M. et al. Amplification of acoustic evanescent waves using metamaterial slabs. Phys. Rev. Lett. 107, 194301 (2011).

16. Zhang, S. et al. Negative refractive index in chiral metamaterials. Phys. Rev. Lett. 102, 023901 (2009).

17. Ahmadi, A. \& Mosallaei, H. Physical configuration and performance modeling of all-dielectric metamaterials. Phys. Rev. B 77, 045104 (2008).

18. Zhang, S. et al. Near-infrared double negative metamaterials. Opt. Express 13, 4922-4930 (2005).

19. Huang, X. et al. Dirac cones induced by accidental degeneracy in photonic crystals and zero-refractive-index materials. Nat. Mater. 10, 582-586 (2011).

20. Soukoulis, C. M. \& Wegener, M. Past achievements and future challenges in the development of three-dimensional photonic metamaterials. Nat. Photon. 5, 523-530 (2011).

21. Li, Y. et al. On-chip zero-index metamaterials. Nat. Photon. 9, 738-742 (2015).

22. Choi, M. et al. A terahertz metamaterial with unnaturally high refractive index. Nature 470, 369-373 (2011).

23. Hu, H., Ji, D., Zeng, X., Liu, K. \& Gan, Q. Rainbow Trapping in Hyperbolic Metamaterial Waveguide. Sci. Rep. 3, 1249 (2013).

24. Mackay, T. G. \& Lakhtakia, A. Electromagnetic Anisotropy and Bianisotropy: A Field Guide (World Scientific, 2010).

25. Li, Z., Aydin, K. \& Ozbay, E. Determination of the effective constitutive parameters of bianisotropic metamaterials from reflection and transmission coefficients. Phys. Rev. E 79, 026610 (2009).

26. Saadoun, M. M. I. \& Engheta, N. A reciprocal phase shifter using novel pseudochiral or $\omega$ medium. Microw. Opt. Technol. Lett. 5, 184-188 (1992).

27. Yu, S., Piao, X., Hong, J. \& Park, N. Bloch-like waves in random-walk potentials based on supersymmetry. Nat. Commun. 6, 8249 (2015).

28. Pendry, J. B., Schurig, D. \& Smith, D. R. Controlling electromagnetic fields. Science 312, 1780-1782 (2006).

29. Schurig, D. et al. Metamaterial electromagnetic cloak at microwave frequencies. Science 314, 977-980 (2006).

30. Ni, X., Kildishev, A. V. \& Shalaev, V. M. Metasurface holograms for visible light. Nat. Commun. 4, 2807 (2013).

31. Driscoll, T. et al. Memory metamaterials. Science 325, 1518-1521 (2009).

32. Zheludev, N. I. \& Kivshar, Y. S. From metamaterials to metadevices. Nat. Mater. 11, 917-924 (2012).

33. Ou, J. Y., Plum, E., Jiang, L. \& Zheludev, N. I. Reconfigurable photonic metamaterials. Nano Lett. 11, 2142-2144 (2011).

34. Alù, A. First-principles homogenization theory for periodic metamaterials. Phys. Rev. B 84, 075153 (2011).

35. Yu, N. et al. Light propagation with phase discontinuities: generalized law of reflection and refraction. Science 334, 333-337 (2011).
36. Sun, S. et al. Gradient-index meta-surfaces as a bridge linking propagating waves and surface waves. Nat. Mater. 11, 426-431 (2012).

37. Ni, X., Emani, N. K., Kildishev, A. V., Boltasseva, A. \& Shalaev, V. M. Broadband light bending with plasmonic nanoantennas. Science 335, 427 (2012).

38. Robillard, J-F. et al. Phononic metamaterials for thermal management: an atomistic computational study. Chinese J. Phys. 49, 448-461 (2011).

39. Oh, J. H., Kwon, Y. E., Lee, H. J. \& Kim, Y. Y. Elastic metamaterials for independent realization of negativity in density and stiffness. Sci. Rep. 6, 23630 (2016).

40. Bückmann, T. et al. An elasto-mechanical unfeelability cloak made of pentamode metamaterials. Nat. Commun. 5, 4130 (2014).

41. Brun, M., Guenneau, S. \& Movchan, A. B. Achieving control of in-plane elastic waves. Appl. Phys. Lett. 94, 061903 (2009).

42. Brûlé, S., Javelaud, E. H., Enoch, S. \& Guenneau, S. Experiments on seismic metamaterials: molding surface waves. Phys. Rev. Lett. 112, 133901 (2014).

43. Ruan, Z. \& Fan, S. Superscattering of light from subwavelength nanostructures Phys. Rev. Lett. 105, 013901 (2010).

44. Kang, J. H., Kim, D. S. \& Park, Q.-H. Local capacitor model for plasmonic electric field enhancement. Phys. Rev. Lett. 102, 093906 (2009).

45. Chakram, S., Patil, Y.S., Chang, L. \& Vengalattore, M. Dissipation in ultrahigh quality factor SiN membrane resonators. Phys. Rev. Lett. 112, 127201 (2014).

46. Kinsler, L. E., Frey, A. R., Coppens, A. B. \& Sanders, J. V. Fundamental of Acoustics (John Wiley \& Sons, 1999).

\section{Acknowledgements}

This study was supported by the National Research Foundation funded by the Ministry of Science, ICT and Future Planning (Global Frontier Program: No. NRF2014M3A6B3063708 and Global Research Laboratory: No. NRF-2008-00580).

\section{Author contributions}

S.K. and N.P. devised the structure of the meta-atom and wrote the manuscript. S.K. performed the theoretical analysis of the meta-atom and designed the experiment. C.C. verified the analytical and numerical results, prepared the experimental set-up and performed measurements. J.J. fabricated the meta-atom structures. N.P. conceived the idea and checked the theoretical derivations. All authors contributed to the revisions of the manuscript.

\section{Additional information}

Supplementary Information accompanies this paper at http://www.nature.com/ naturecommunications

Competing financial interests: The authors declare no competing financial interests.

Reprints and permission information is available online at http://npg.nature.com/ reprintsandpermissions/

How to cite this article: Koo, S. et al. Acoustic omni meta-atom for decoupled access to all octants of a wave parameter space. Nat. Commun. 7, 13012 doi: $10.1038 /$ ncomms13012 (2016).

This work is licensed under a Creative Commons Attribution 4.0 International License. The images or other third party material in this article are included in the article's Creative Commons license, unless indicated otherwise in the credit line; if the material is not included under the Creative Commons license, users will need to obtain permission from the license holder to reproduce the material. To view a copy of this license, visit http://creativecommons.org/licenses/by/4.0/

(C) The Author(s) 2016 